\title{
Critical loads of built-up columns and a pocket calculator program
}

\author{
D. M. PORTER \& F. W. WILLIAMS
}

\section{Mr R. C. Obbard, $M$}

The theory presented assumes rigid battens and that 'no shear' stability functions ${ }^{2}$ are appropriate. Such assumptions are justified if they lead to useful practical solutions, of adequate accuracy, which can be obtained on a pocket calculator.

34. The rigid batten assumption is a major restriction on the usefulness of the theory. Before any designer can use the theory presented with confidence, it is essential to define when the theory is, and is not, appropriate. It would have been helpful if the Authors had compared their results with results from a standard second order frame program, and then attempted to define the limits within which their theory was appropriate. Their comparison with a special case of the equation due to Timoshenko and Gere ${ }^{1}$ is not enlightening in this respect.

35. I suggest that a test based on the equation due to Timoshenko and Gere should be used to check whether or not the proposed theory will yield answers of adequate accuracy.

36. I have used the equation due to Timoshenko and Gere for a number of years in connection with the estimation of collapse loads for falsework and scaffolding assemblies, and I believe it produces good practical solutions for a wide range of structural systems. Its usefulness is not confined to built-up columns.

37. Equation (1) is derived from the result

$$
P_{\mathrm{er}}=\frac{\pi^{2} E I_{\mathrm{t}} / L^{2}}{1+\left(\pi^{2} E I_{\mathrm{t}} / L^{2} k_{\mathrm{sh}}\right)}
$$

where $k_{\mathbf{s h}}$ is the shear stiffness for the structure/strut and is defined in Fig. 9.

38. Theoretical values of $k_{\mathrm{sh}}$ can be substituted into equation (12) for normal truss and frame systems. For complex arrangements $k_{\mathrm{sh}}$ may be obtained by experiment. For frame systems and battened struts (Fig. 10) $k_{\mathrm{sh}}$ is normally calculated from

$$
\frac{1}{k_{\mathrm{ah}}}=\frac{l_{\mathrm{p}}}{12 E \sum\left(I_{\mathrm{r}} / I_{\mathrm{p}}\right)}+\frac{l_{\mathrm{p}}}{12 E \sum\left(I_{\mathrm{b}} / I_{\mathrm{b}}\right)} \quad . \quad \text {. . . . . . }
$$

For the special case of a battened strut made up from two identical main sections (as is normal), equation (12) then becomes

$$
P_{\mathrm{cr}}=\frac{\pi^{2} E I_{\mathrm{t}} / L^{2}}{1+\left(\pi^{2} E I_{\mathrm{l}} / L^{2}\right)\left[\left(l_{\mathrm{D}}^{2} / 24 E I_{\mathrm{f}}\right)+\left(l_{\mathrm{p}} l_{\mathrm{b}} / 12 E I_{\mathrm{b}}\right)\right]} \quad \cdot \quad \cdot \quad \cdot .
$$

For the even more restricted case where the battens are assumed to be rigid equation (12) reduces to equation (1). 
Fig. 9
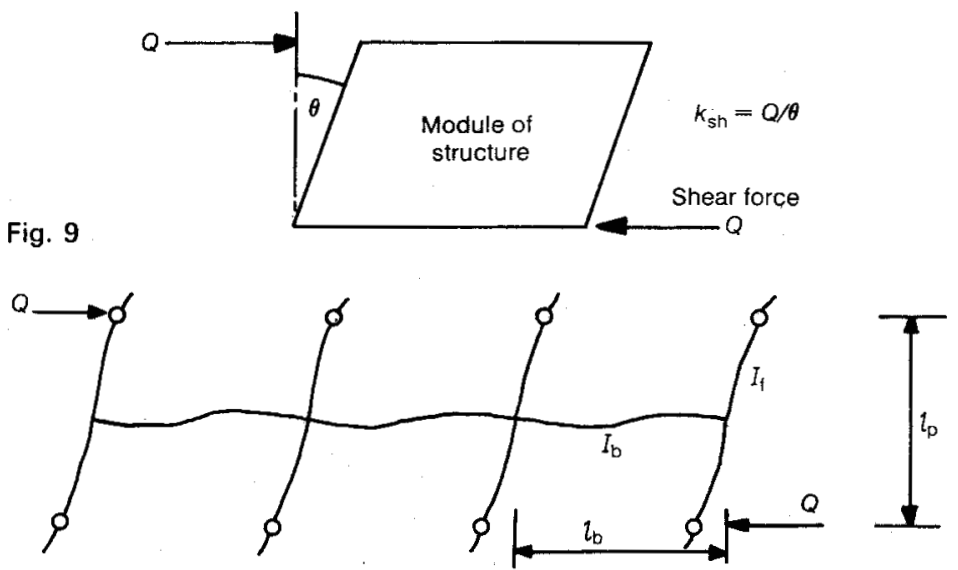

Fig. 10

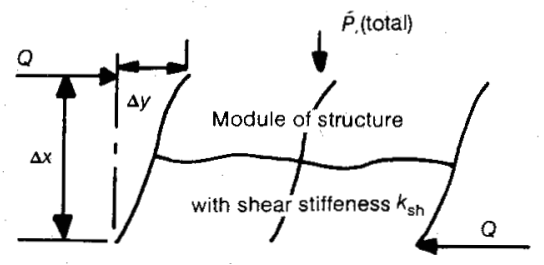

Fig. 11

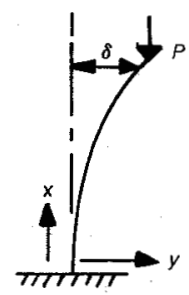

Fig. 12

39. Rearranging equation (12) and substituting from equation (13) gives

$$
P_{\mathrm{cr}}=\left[\frac{L^{2}}{\pi^{2} E I_{\mathrm{t}}}+\frac{l_{\mathrm{p}}}{12 E \sum\left(I_{\mathrm{f}} / l_{\mathrm{p}}\right)}+\frac{l_{\mathrm{p}}}{12 E \sum\left(I_{\mathrm{b}} / I_{\mathrm{b}}\right)}\right]^{-1}
$$

in which the first term on the right-hand side is due to axial loads in columns, the second is due to bending of columns and the third is due to bending of battens.

40. For medium-rise frame building, scaffolding assemblies, and similar structures, the term due to axial loading is often negligible in comparison with the other two terms. Hence the collapse load of a frame system is defined for the sidesway collapse mode by

$$
P_{\mathrm{cr}}=k_{\mathrm{sh}} \text {. . . . . . . . . . . . . }
$$

This is a very useful form of equation (12) and provides good ball park solutions for the collapse of frame systems with incredible simplicity.

41. If axial shortening is ignored then the derivation of equation (12) given by Timoshenko and Gere ${ }^{1}$ points to the solution

$$
\Delta y=\Delta x\left[\frac{Q}{k_{\mathrm{sh}}} /\left(1-\frac{P}{k_{\mathrm{sh}}}\right)\right] \quad . \quad . \quad . \quad . \quad . \quad . \quad .
$$

where $\Delta x$ and $\Delta y$ are as defined in Fig. 11 .

42. This simple equation gives good deflexion solutions for frames. I have checked deflexions derived using equation (17) against the second order solutions of Cheong Siat $\mathrm{Moy}^{8}$ and found both sets of solutions virtually identical. 
43. As equation (15) appears to produce good results (for $u \geqslant 6$ ) for the collapse/ deflexion of structural systems as diverse as multi-bay frames and battened struts, it must include all the relevant variables. The difficulty with the theoretical method proposed in the Paper is that batten stiffness cannot be considered. Equation (15) indicates that if the battens have a stiffness of the same order of magnitude as the columns, then they will have twice the effect of the columns on the collapse load of the system. Such an effect cannot be ignored.

44. Assuming that an approximate solution is satisfactory if it provides answers within $10 \%$, then from equation (15) the method of analysis proposed in the Paper should be adequate provided

$$
\frac{l_{\mathrm{p}}}{12 E \sum\left(I_{\mathrm{b}} / l_{\mathrm{b}}\right)} /\left[\frac{L^{2}}{\pi^{2} E I_{\mathrm{t}}}+\frac{l_{\mathrm{p}}}{12 E \sum\left(I_{\mathrm{t}} / l_{\mathrm{p}}\right)}\right] \leqslant 0.1 . . . . .
$$

45. It may well be worth using the proposed program for non-uniform battened struts, provided the condition in equation (18) is met. However, such a condition is very restricting.

46. If the Authors were to modify their theory to allow for flexure of the battens, the program would be far more useful. Unfortunately, batten flexure is not simple to deal with because the relationship between axial shortening and rotation of ends of the members is invalidated.

47. Equation (12) gives good results for uniform struts. It should be possible to deal with non-uniform struts by incremental solution of the basic differential equation from which it is derived (see Fig. 12), i.e.

$$
\frac{\mathrm{d}^{2} y}{\mathrm{~d} x^{2}}=\left\{\frac{P}{E I_{\mathrm{t}}\left[1-\left(P / k_{\mathrm{nb}}\right)\right]}\right\}(\delta-y) \quad . \quad . \quad . \quad . \quad . \quad .
$$

48. If the Authors can get such a solution on a calculator program, then they will be able to get reasonable results for braced and unbraced multi-bay frames, and battened and laced struts.

\section{Mr Porter and Professor Williams}

The range in which connectors can be treated as rigid was not defined in the Paper because such definition would have been lengthy, due to the number of parameters involved. This question was therefore left to the engineer's judgement. The discussion should help the engineer to develop such judgement and also to decide when the various simple equations which give ball park solutions are sufficient. However, the repeated references to frames could be misleading as the Paper is explicitly restricted to built-up columns.

50. We believe that the assumption of rigid connectors is much more frequently valid for the range of battened columns envisaged in BS 449 than the restrictive comment at the end of $\$ 45$ suggests. This difference of opinion arises because we judge that conditions such as equation (18) will be satisfied frequently, and that such conditions are unduly restrictive because the large size of the connections between the vertical components and connectors makes the connector stiffness considerably greater than is implied by the term $12 E \Sigma\left(I_{\mathrm{b}} / l_{\mathrm{b}}\right)$ in equation (18) (also, see $\$ 18$ ).

51. Equation (15) is only approximate, and can be misleading. Hence it gives $P_{\mathrm{cr}} \rightarrow 0$ as $I_{\mathrm{b}} \rightarrow 0$, whereas when the connector stiffness is negligible the correct answer is $P_{\text {ar }}=\pi^{2} E \Sigma I_{\mathrm{r}} / L^{2}$.

\section{Reference}

8. Cheong Siat Moy F. Control of deflexions in unbraced steel frames. Proc. Instn Civ. Engrs, Part 2, 1974, 57, Dec., 619-634. 